\title{
Facile Synthesis of Monodispersed Ag NPs in Ethylene Glycol Using Mixed Capping Agents
}

\author{
Suyue Chen, Jacob R. Drehmel, and R. Lee Penn*
}

Department of Chemistry, University of Minnesota, 207 Pleasant Street SE, Minneapolis, MN 55455, USA 
Table S1. Amounts of capping reagents used in $5 \mathrm{~mL} \mathrm{Ag} \mathrm{NPs} \mathrm{syntheses.}$

\begin{tabular}{|c|c|c|c|c|c|}
\hline \multicolumn{3}{|c|}{ Target Concentrations $(\mu \mathrm{M})$} & \multicolumn{3}{|c|}{ Capping Agent Solutions Added $(\mu \mathrm{L} \times \mathbf{m M})$} \\
\hline $\mathbf{P V P}^{1}$ & TA & Citrate & PVP ${ }^{1}$ & TA & $\begin{array}{l}\text { Sodium } \\
\text { Citrate }\end{array}$ \\
\hline 1000 & 1 & 0 & $100 \times 50$ & $1.6 \times 3.12(5)$ & 0 \\
\hline 1000 & 5 & 0 & $100 \times 50$ & $1.6 \times 15.62(5)$ & 0 \\
\hline 1000 & 10 & 0 & $100 \times 50$ & $1.6 \times 31.25$ & 0 \\
\hline 1000 & 50 & 0 & $100 \times 50$ & $8 \times 31.25$ & 0 \\
\hline 1000 & 100 & 0 & $100 \times 50$ & $16 \times 31.25$ & 0 \\
\hline 1000 & 150 & 0 & $100 \times 50$ & $24 \times 31.25$ & 0 \\
\hline 1000 & 1000 & 0 & $100 \times 50$ & $160 \times 31.25$ & 0 \\
\hline 1000 & 0 & 0 & $100 \times 50$ & 0 & 0 \\
\hline 2000 & 0 & 0 & $200 \times 50$ & 0 & 0 \\
\hline 3000 & 0 & 0 & $300 \times 50$ & 0 & 0 \\
\hline 4000 & 0 & 0 & $400 \times 50$ & 0 & 0 \\
\hline 0 & 1 & 0 & 0 & $1.6 \times 3.12(5)$ & 0 \\
\hline 0 & 10 & 0 & 0 & $1.6 \times 31.25$ & 0 \\
\hline 0 & 100 & 0 & 0 & $16 \times 31.25$ & 0 \\
\hline 0 & 1000 & 0 & 0 & $160 \times 31.25$ & 0 \\
\hline 1000 & 0 & 10 & $100 \times 50$ & 0 & $125 \times 0.4$ \\
\hline 1000 & 0 & 100 & $100 \times 50$ & 0 & $125 \times 4$ \\
\hline 1000 & 0 & 1000 & $100 \times 50$ & 0 & $125 \times 40$ \\
\hline 100 & 0 & 1000 & $100 \times 5$ & 0 & $125 \times 40$ \\
\hline 10 & 0 & 1000 & $100 \times 0.5$ & 0 & $125 \times 40$ \\
\hline 0 & 0 & 1000 & 0 & 0 & $125 \times 40$ \\
\hline
\end{tabular}

${ }^{1}$ concentration by repeat units 
Table S2. Amounts of capping reagents used in $25 \mathrm{~mL} \mathrm{Ag} \mathrm{NPs} \mathrm{syntheses.}$

\begin{tabular}{cc|cc}
\hline \multicolumn{2}{c|}{ Target Concentrations $(\boldsymbol{\mu M})$} & \multicolumn{2}{|c}{ Capping Agent Solutions Added $(\boldsymbol{\mu L} \times \mathbf{m M})$} \\
\hline PVP $^{1}$ & TA & PVP & TA \\
\hline 1000 & 0 & $500 \times 50$ & 0 \\
1000 & 1 & $500 \times 50$ & $8 \times 3.12(5)$ \\
1000 & 10 & $500 \times 50$ & $80 \times 3.12(5)$ \\
1000 & 100 & $500 \times 50$ & $80 \times 31.25$ \\
0 & 1 & 0 & $8 \times 3.12(5)$ \\
0 & 10 & 0 & $80 \times 3.12(5)$ \\
0 & 100 & 0 & $80 \times 31.25$ \\
\hline
\end{tabular}

${ }^{1}$ concentration by repeat units 

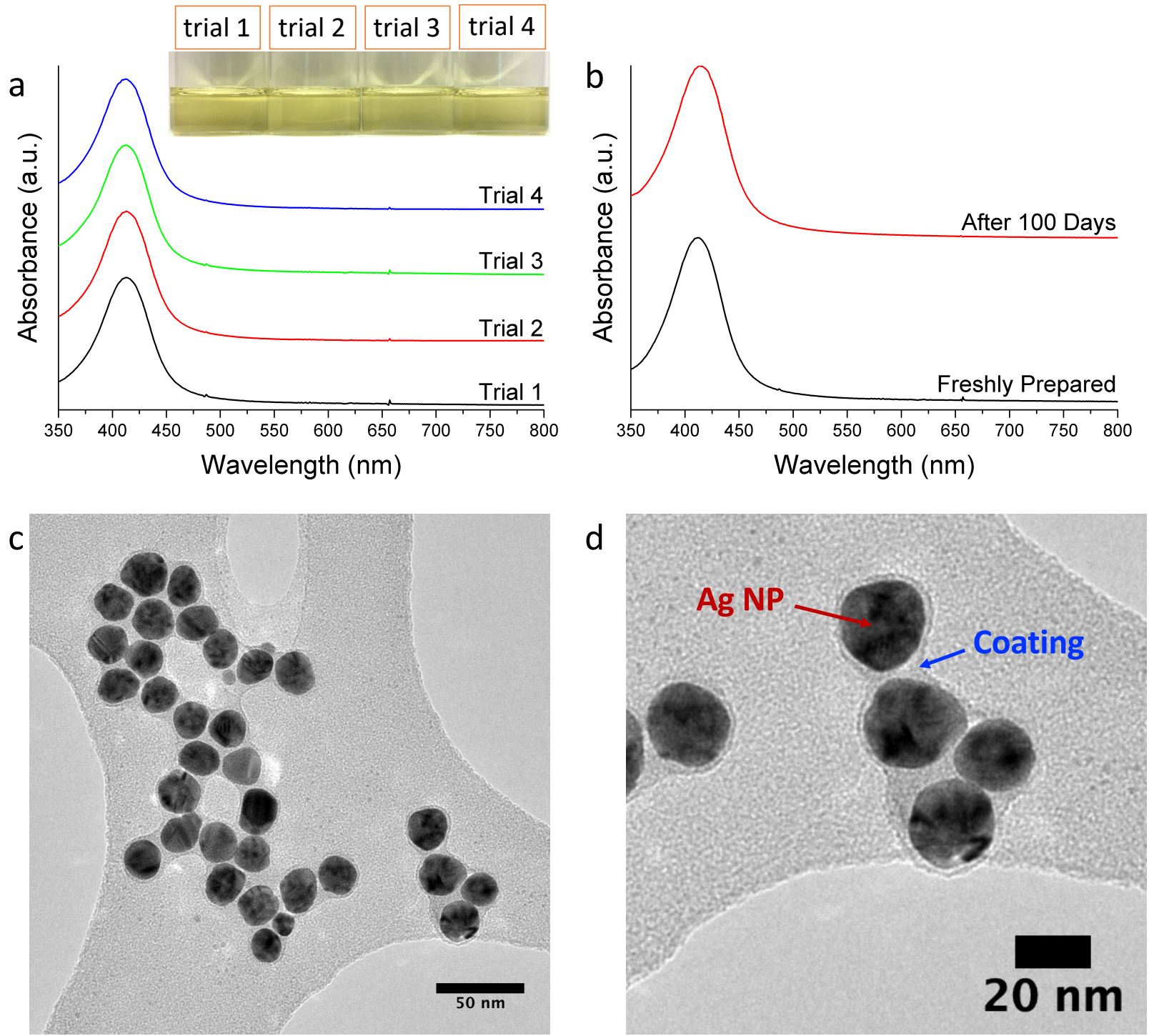

Figure S1. UV-vis spectra of (a) as-prepared reaction mixtures from four trials of polyol reactions at $150{ }^{\circ} \mathrm{C}$ using $30 \mu \mathrm{M} \mathrm{AgOCOCF}, 1,1 \mathrm{mM} \mathrm{PVP}$ and $100 \mu \mathrm{M} \mathrm{TA}$ and (b) a reaction mixture freshly prepared and after stored at ca. $4{ }^{\circ} \mathrm{C}$ for 100 days. Each spectrum is normalized to its highest peak. Image (c) is a representative TEM image of the Ag NPs in reaction mixture after storage at $4{ }^{\circ} \mathrm{C}$ for 100 days. Image (d) showed a zoomed-in TEM image of Ag NPs illustrate how the polymer coating on Ag NPs can be observed in TEM images. Insets in (a) are photographs of reaction mixtures in $20 \mathrm{~mL}$ scintillation vials. 


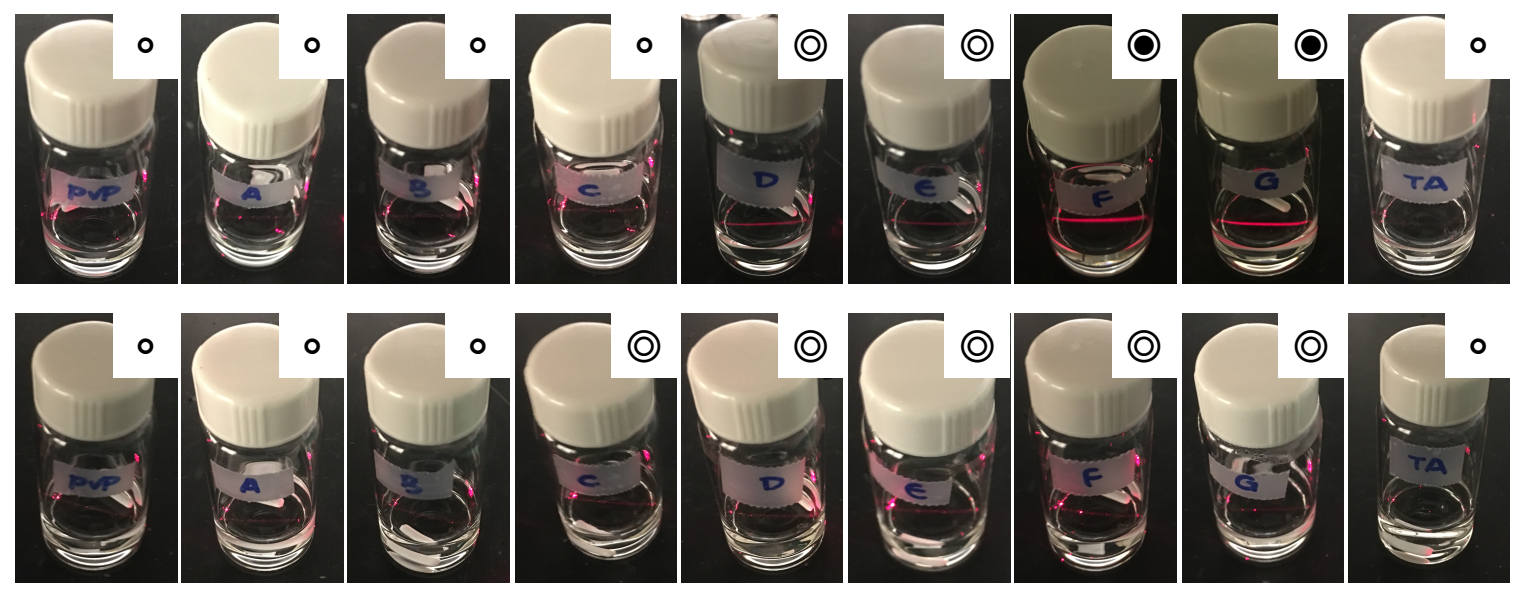

Figure S2. Photographs of reaction mixtures during the laser scattering test. The PVP concentration was $1 \mathrm{mM}$ in the vial marked as PVP and vials A-G, and $0 \mathrm{mM}$ in the vial marked as TA. TA concentration varied: (PVP) $0 \mu \mathrm{M}$, (A) $1 \mu \mathrm{M}$, (B) $5 \mu \mathrm{M}$, (C) $10 \mu \mathrm{M}$, (D) $50 \mu \mathrm{M}$, (E) $100 \mu \mathrm{M},(\mathbf{F}) 500 \mu \mathrm{M},(\mathbf{G}$ and TA) $1 \mathrm{mM}$. The photographs for the top row were collected at room temperature and bottom row after heated to $150{ }^{\circ} \mathrm{C}$. Insets indicate the relative strength of the observed scattering: $\bullet$ little to no scattering, $\odot$ weak scattering, and $\odot$ strong scattering. 


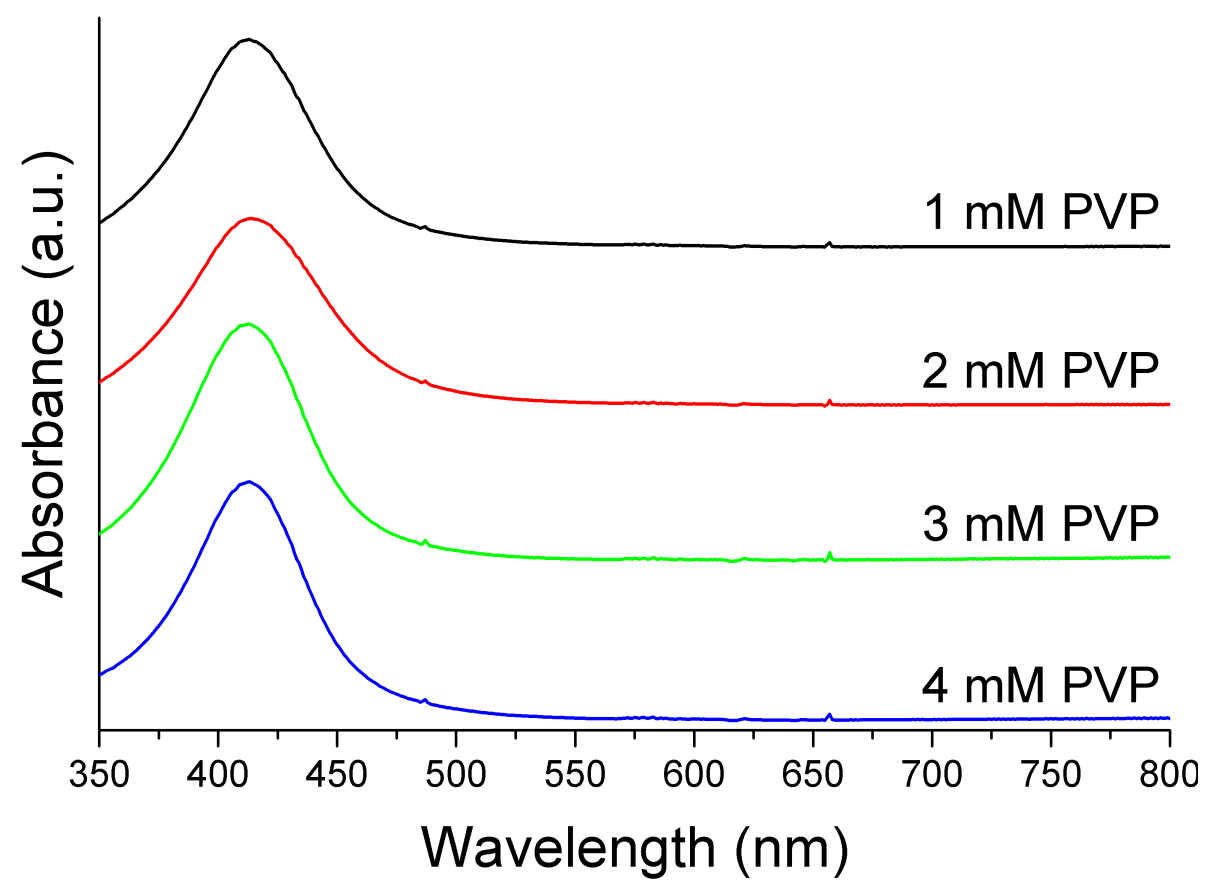

Figure S3. UV-vis spectra of as prepared product mixtures from polyol reactions at $150{ }^{\circ} \mathrm{C}$ using $30 \mu \mathrm{M} \mathrm{AgOCOCF} 3$ and different concentrations of PVP. Each spectrum is normalized to its highest peak. 


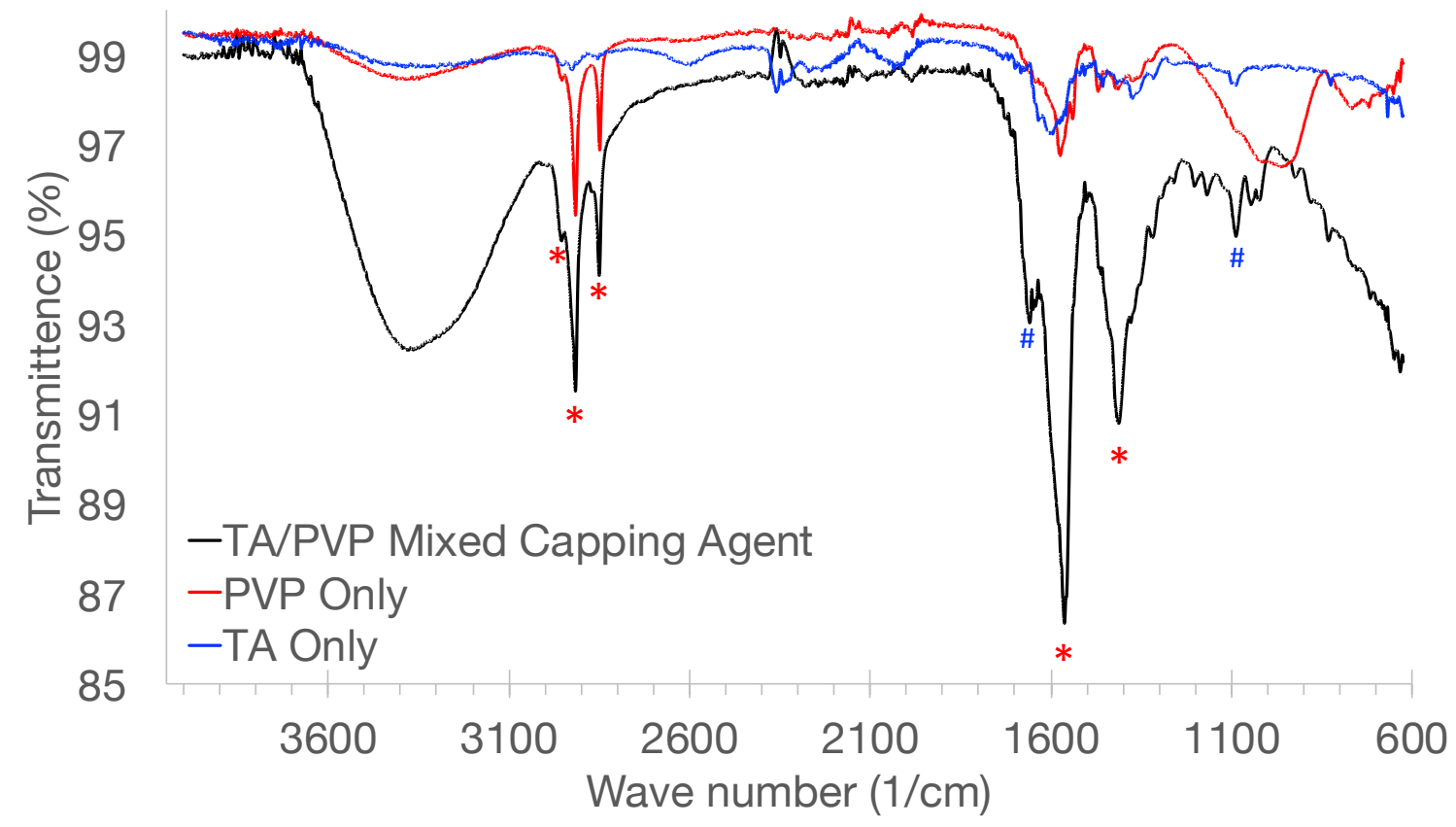

Figure S4. Attenuated total reflection - Fourier-transform infrared (ATR-FTIR) spectra of Ag NPs with different organic coating synthesized from polyol reactions at $150{ }^{\circ} \mathrm{C}$ using $30 \mu \mathrm{M}$ $\mathrm{AgOCOCF}_{3}$ and (red) $1 \mathrm{mM}$ PVP, (blue) $1 \mathrm{mM} \mathrm{TA}$, or (black) $1 \mathrm{mM}$ PVP and $100 \mu \mathrm{M}$ TA. The black spectrum shows peaks correlating to the blue spectrum (at $1658 \mathrm{~cm}^{-1}$ and $1087 \mathrm{~cm}^{-1}$, marked by \#), as well as peaks correlating to the red spectrum (at $2941 \mathrm{~cm}^{-1}, 2915 \mathrm{~cm}^{-1}, 2850 \mathrm{~cm}^{-1}, 1562$ $\mathrm{cm}^{-1}$, and $1411 \mathrm{~cm}^{-1}$, marked by *). 

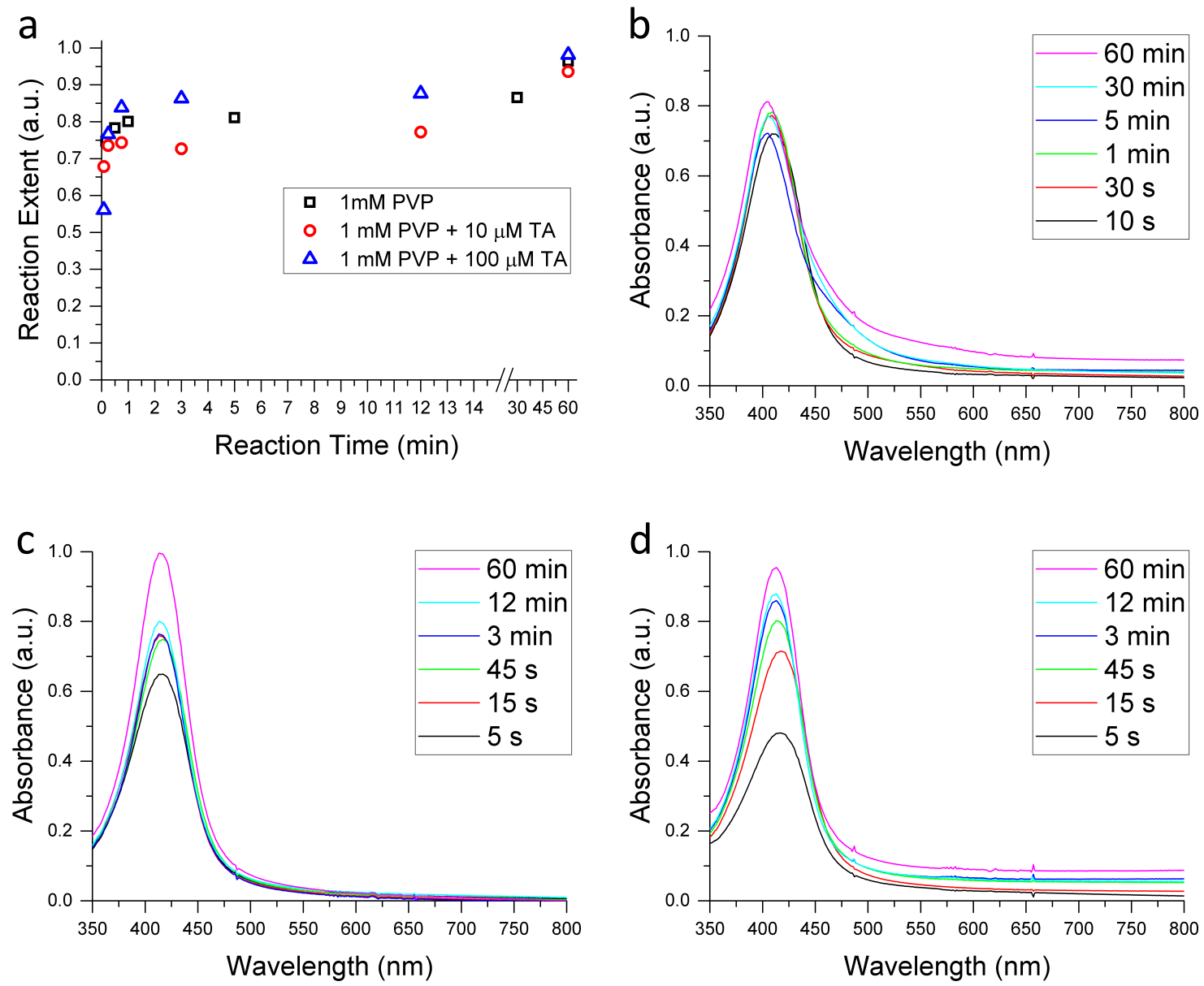

Figure S5. (a) Plot of reaction extent and (b-d) UV-vis spectra over time for polyol reactions (each with $5 \mathrm{~mL}$ total volume) at $150{ }^{\circ} \mathrm{C}$ using $30 \mu \mathrm{M} \mathrm{AgOCOCF} 3$ and varied concentrations of PVP and TA: (b) $1 \mathrm{mM}$ PVP, (c) $1 \mathrm{mM}$ PVP and $10 \mu \mathrm{M}$ TA, and (d) $1 \mathrm{mM}$ PVP and $100 \mu \mathrm{M}$ TA. The reaction extent of each sample was calculated by dividing the absorbance peak area between 350 $\mathrm{nm}$ and $550 \mathrm{~nm}$ of the corresponding UV-vis spectrum by the that of the 60 min sample from the reaction using $1 \mathrm{mM}$ PVP and $10 \mu \mathrm{M}$ TA (c). All spectra in (b-d) are normalized to the peak height of the 60 min sample in (c). 

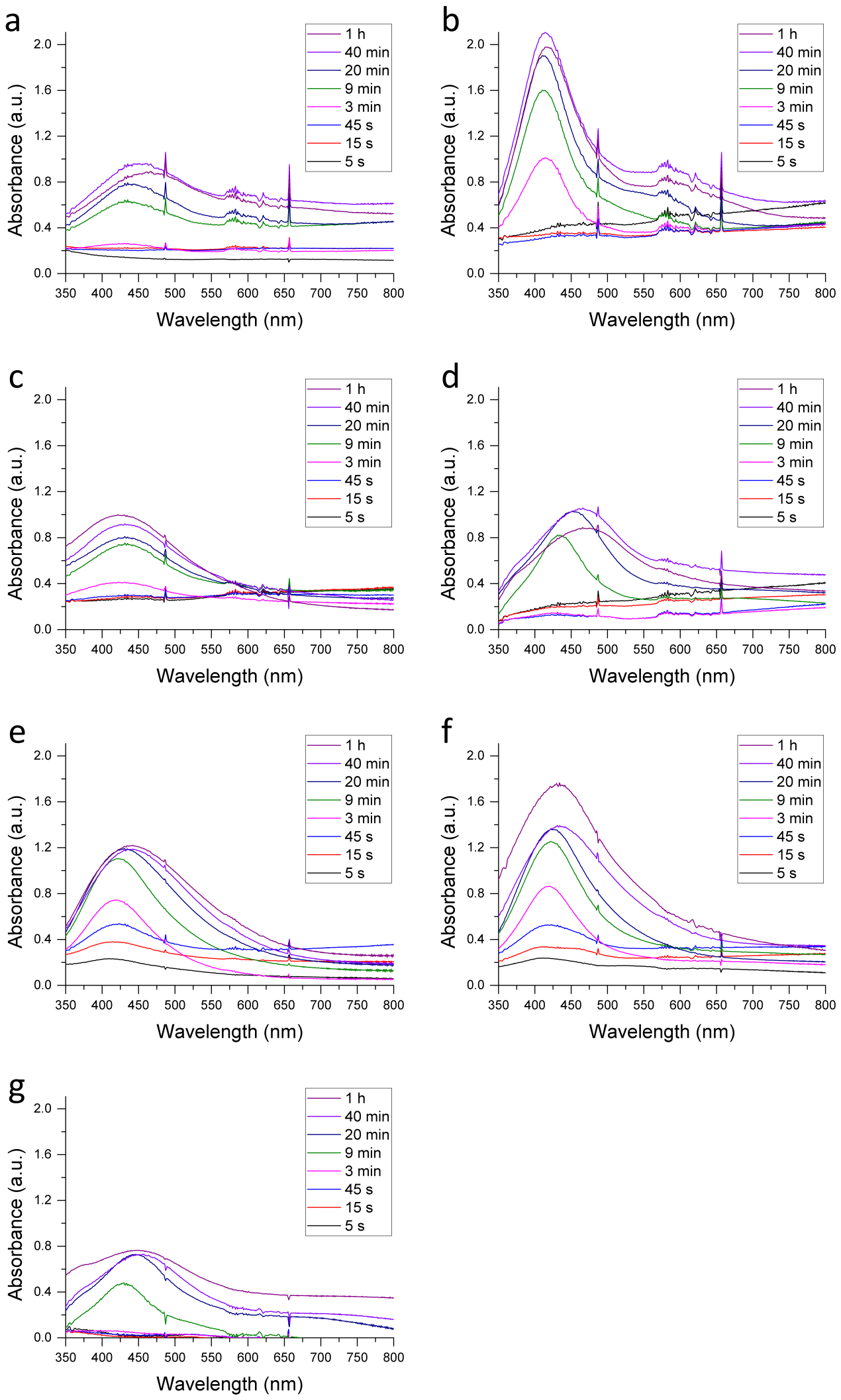
Figure S6. UV-vis spectra of as-prepared reaction mixtures over time from scaled-up polyol reactions ( $25 \mathrm{~mL}$ in total volume) at $150{ }^{\circ} \mathrm{C}$ using $30 \mu \mathrm{M} \mathrm{AgOCOCF} 3$ and varied concentrations of PVP and TA: (a) $1 \mathrm{mM} \mathrm{PVP,} \mathrm{(b)} 1 \mathrm{mM}$ PVP and $1 \mu \mathrm{M} \mathrm{TA}$, (c) $1 \mathrm{mM} \mathrm{PVP}$ and $10 \mu \mathrm{M} \mathrm{TA}$, (d) $1 \mathrm{mM}$ PVP and $100 \mu \mathrm{M} \mathrm{TA}$, (e) $1 \mu \mathrm{M} \mathrm{TA}$, (f) $10 \mu \mathrm{M} \mathrm{TA}$, (g) $100 \mu \mathrm{M}$ TA. All spectra are normalized to the $60 \mathrm{~min}$ sample from the reaction using $1 \mathrm{mM} \mathrm{PVP}$ and $10 \mu \mathrm{M} \mathrm{TA}$. 


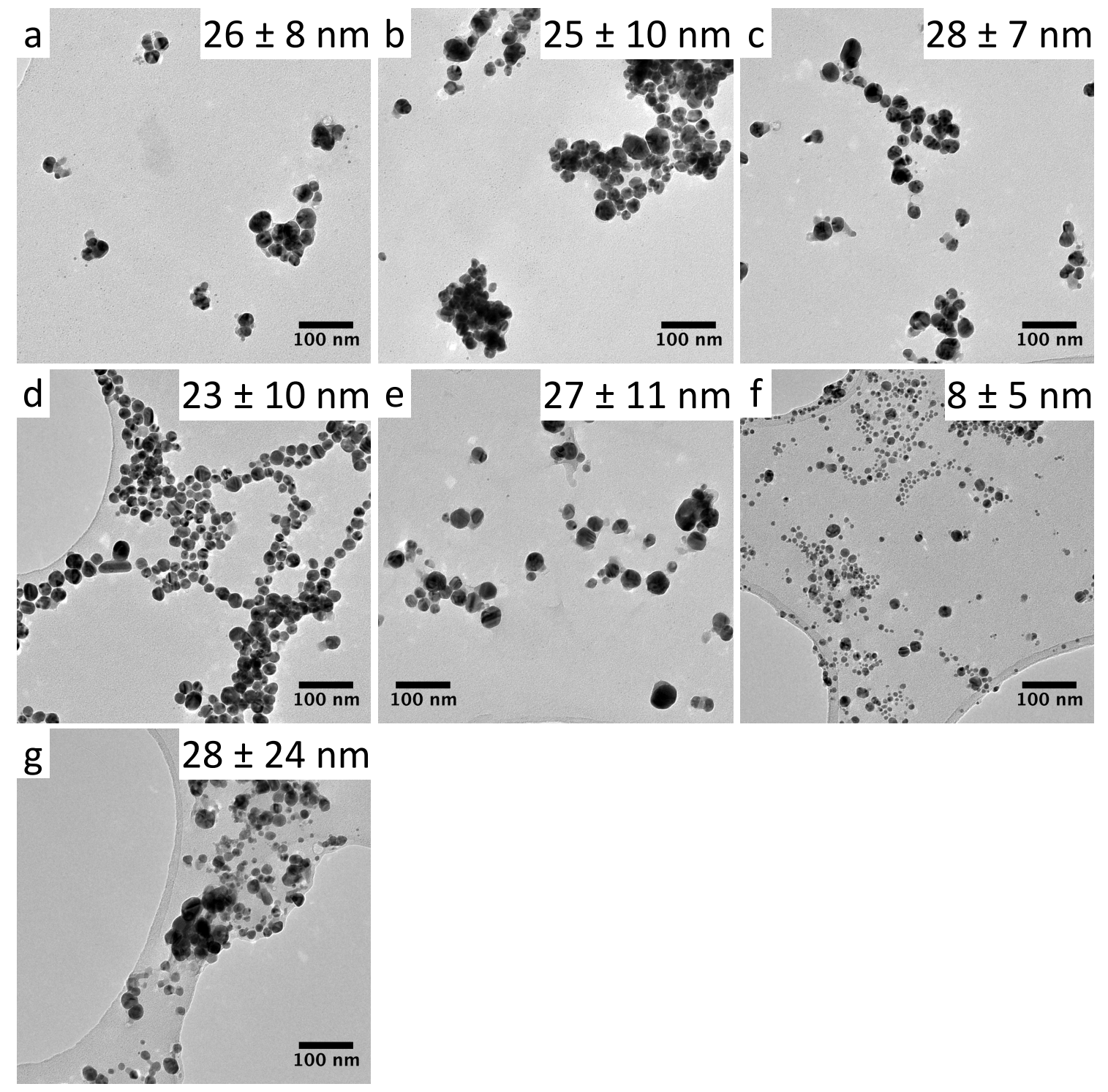

Figure S7. Representative TEM images of Ag NPs collected from polyol reactions at $150{ }^{\circ} \mathrm{C}$ using $30 \mu \mathrm{M} \mathrm{AgOCOCF} 3$ and varied concentrations of PVP and sodium citrate: (a) $1 \mathrm{mM} \mathrm{PVP,} \mathrm{(b)} 1$ $\mathrm{mM}$ PVP and $10 \mu \mathrm{M}$ sodium citrate, (c) $1 \mathrm{mM}$ PVP and $100 \mu \mathrm{M}$ sodium citrate, (d) $1 \mathrm{mM}$ PVP and $1 \mathrm{mM}$ sodium citrate, (e) $100 \mu \mathrm{M}$ PVP and $1 \mathrm{mM}$ sodium citrate, (f) $10 \mu \mathrm{M}$ PVP and $1 \mathrm{mM}$ sodium citrate, and (g) $1 \mathrm{mM}$ sodium citrate. Average Ag NP diameters and standard deviations are marked in each image. 